\title{
Post-Newtonian gravitational radiation and equations of motion via direct integration of the relaxed Einstein equations. V. Evidence for the strong equivalence principle to second post-Newtonian order
}

\author{
Thomas Mitchell and Clifford M. Will $*$ \\ McDonnell Center for the Space Sciences, Department of Physics, \\ Washington University, St. Louis, Missouri 63130
}

(Dated: September 15, 2021)

\begin{abstract}
Using post-Newtonian equations of motion for fluid bodies valid to the second post-Newtonian order, we derive the equations of motion for binary systems with finite-sized, non-spinning but arbitrarily shaped bodies. In particular we study the contributions of the internal structure of the bodies (such as self-gravity) that would diverge if the size of the bodies were to shrink to zero. Using a set of virial relations accurate to the first post-Newtonian order that reflect the stationarity of each body, and redefining the masses to include 1PN and 2PN self-gravity terms, we demonstrate the complete cancellation of a class of potentially divergent, structure-dependent terms that scale as $s^{-1}$ and $s^{-5 / 2}$, where $s$ is the characteristic size of the bodies. This is further evidence of the Strong Equivalence Principle, and supports the use of post-Newtonian approximations to derive equations of motion for strong-field bodies such as neutron stars and black holes. This extends earlier work done by Kopeikin.
\end{abstract}

\section{INTRODUCTION AND SUMMARY}

The principle of equivalence is the cornerstone of Einstein's general theory of relativity. Part of this principle, known as the weak equivalence principle (WEP) states that test bodies fall in a gravitational field with the same acceleration, irrespective of their internal structure or composition. By test body, one means a body whose internal structure is governed by non-gravitational interactions, and whose size is small compared to inhomogeneities in external gravitational fields (suppression of tidal couplings). WEP, together with postulates of local Lorentz invariance and local position invariance of non-gravitational physics in freely falling frames, comprises the Einstein Equivalence Principle (EEP), which is the foundation of metric gravity, or of the idea that gravity is really geometry.

There is a stronger version of WEP, which proposes that all "test" bodies should fall with the same acceleration, even bodies that are self-gravitating, such as stars, planets, or black holes. Here, by test body, one means a body that is large and massive enough to have measurable self-gravity, yet is small enough that tidal interactions can be ignored (one generally ignores spin interactions as well). This version of WEP is a part of the Strong Equivalence Principle (SEP), which includes a postulate of a kind of local Lorentz invariance and position invariance of gravity itself. While every metric theory of gravity obeys EEP, almost no metric theory obeys SEP. Scalar-tensor theories of gravity, such as the Brans-Dicke theory and its extensions, violate SEP. Indeed, SEP is generically violated in theories of gravity that introduce long-range fields, in addition to the spacetime metric, that mediate how the metric is generated by matter. In order to preserve EEP, such fields do not couple directly to matter. Because general relativity contains one and only one long-range gravitational field - the metric itself - it has no mechanism for violating SEP. Far from any gravitating system, the metric can always be made suitably, if approximately, Minkowskian, and so there is no obvious mechanism, other than tidal interactions, for the external universe, or for any nearby "spectator" body to influence the internal structure or dynamics of the system (for further details of this argument, see [1] and Sec. 3.3 of [2]). Thus gravity in GR is independent of the velocity of the system relative to some external frame, the effective constant of gravity, $G$, is a true constant, and self-gravitating non-spinning bodies move as if they were test particles (for further discussion of SEP, see Section 3.3 of [2]; for an alternative discussion, see [3]).

But these are general, qualitative arguments. This paper addresses the question: how explicitly does general relativity manage to satisfy SEP for self-gravitating bodies, specificially to the second order in a post-Newtonian expansion?

At the first post-Newtonian (1PN) order of approximation, that is, at order $\epsilon \sim(v / c)^{2} \sim G m / r c^{2}$ beyond Newtonian gravity, GR has been shown to obey SEP explicitly. This is seen most graphically within the parametrized postNewtonian (PPN) framework, which characterizes the post-Newtonian limit of a wide range of metric theories of gravity using 10 arbitrary parameters (see Chapter 4 of [2] for a review). Some of these parameters or combinations of

*cmw@wuphys.wustl.edu, wugrav.wustl.edu/people/CMW 
them measure whether the theory has "preferred-frame" effects at post-Newtonian order (violations of local Lorentz invariance), some measure whether the locally measured gravitational constant can depend on the presence of nearby matter (violations of local position invariance), and some measure whether self-gravitating bodies violate WEP, a phenomenon known as the Nordtvedt effect. In GR all such offending parameters or combinations of parameters vanish. Furthermore, there is experimental evidence that they vanish from a variety of tests of post-Newtonian gravity, spanning lunar laser ranging, geophysical measurements and binary pulsar data (see [4] for a review).

But what about beyond post-Newtonian order? Does the motion of a pair of finite sized, gravitationally bound, non-spinning bodies depend on their internal structure, apart from tidal interactions? There is existing theoretical evidence that they do not. Breuer and Rudolf [5] showed that the relative "Newtonian-like" acceleration of two well separated bodies, momentarily at rest, was independent of their internal structure, to second post-Newtonian (2PN) order, or order $\epsilon^{2}$ beyond Newtonian gravity; put differently, they proved the absence of the Nordtvedt effect to that order. Kopeikin [6] derived the equations of motion to $2 \mathrm{PN}$ order for self-gravitating bodies with an equation of state $p(\rho)$, under the assumptions that they were spherically symmetric in a suitable comoving frame, and had no internal fluid motions, and also found that the SEP held (see also [7]).

This question is of more than academic interest. The leading candidate sources for gravitational radiation that may be detected in the near future by laser interferometers are binary systems containing neutron stars or black holes. The inspiral part of their evolution can only be described accurately by the post-Newtonian approximation, which, by its very nature, assumes that gravitational fields are weak everywhere in spacetime. No attempt has ever been made to derive the motion to high orders in a PN expansion with either strong-gravity neutron stars or black holes. The closest one has come is to treat the sources as distributions in spacetime (delta functions) and to employ suitable regularization techniques to control the infinities [8, 9], or to use a variant of the surface integral approach pioneered by Einstein, Infeld and Hoffmann [10]. Therefore an explicit demonstration that the internal structure is irrelevant for the binary motion, even if only at $2 \mathrm{PN}$ order, would be valuable.

In this paper we derive the equations of motion of two arbitrarily shaped, non-spinning, gravitationally bound bodies, through 2PN order. Beginning with the $2 \mathrm{PN}$ hydrodynamic equations of motion derived in Paper I of this series [11], we calculate the acceleration of the center of "baryonic" mass of a given body in the field of a companion body. Expanding all variables about the centers of mass of each body we track all terms that scale with the sizes $s$ of the bodies as $s^{-1}$, and $s^{-5 / 2}$; such terms represent contributions due to self-gravitational binding energy, and a kind of gravitomagnetic internal energy, respectively. (In Paper II [12] we ignored all finite-size effects in deriving binary equations of motion.) We then employ virial relations that reflect the equilibrium state of each body, leading to a cancellation of many terms. Finally we renormalize the masses to include both rest mass and gravitational binding energy, by defining the total mass for body A to be

$$
M_{A} \equiv m_{A}+\frac{1}{2} \hat{\Omega}_{A}+O\left(m_{A} \epsilon^{2}\right),
$$

where $m_{A}=\int_{A} \rho^{*} d^{3} x$ is the conserved baryonic mass of body $\mathrm{A}$ and

$$
\hat{\Omega}_{A} \equiv-\frac{1}{2} \int_{A} \int_{A} \frac{\hat{\rho}^{*} \hat{\rho}^{* \prime}}{\left|\hat{\mathbf{x}}-\hat{\mathbf{x}}^{\prime}\right|} d^{3} \hat{x} d^{3} \hat{x}^{\prime}
$$

is the gravitational self-energy as measured in a local, freely falling frame $\hat{x}^{\alpha}$, momentarily comoving with body A. The result is that all self-gravity terms that scale as $s^{-1}$ or $s^{-5 / 2}$ cancel, leaving equations of motion at $2 \mathrm{PN}$ order for body 1 that depend on the renormalized masses $M_{A}$, given by

$$
a_{1}^{i}=-\frac{M_{2}}{r^{2}} n^{i}+a_{1(1 P N)}^{i}+a_{1(2 P N)}^{i},
$$

where $r=\left|\mathbf{x}_{1}-\mathbf{x}_{2}\right|, n^{i}=\left(x_{1}-x_{2}\right)^{i} / r$, and where

$$
\begin{aligned}
a_{1(1 P N)=}^{i} & \frac{M_{2}}{r^{2}} n^{i}\left[4 \frac{M_{2}}{r}+5 \frac{M_{1}}{r}-v_{1}^{2}+4 \mathbf{v}_{1} \cdot \mathbf{v}_{2}-2 v_{2}^{2}+\frac{3}{2}\left(\mathbf{v}_{2} \cdot \mathbf{n}\right)^{2}\right] \\
& +\frac{M_{2}}{r^{2}}\left(v_{1}-v_{2}\right)^{i}\left[4\left(\mathbf{v}_{1} \cdot \mathbf{n}\right)-3\left(\mathbf{v}_{2} \cdot \mathbf{n}\right)\right] \\
a_{1(2 P N)=}^{i} & \frac{M_{2}}{r^{2}} n^{i}\left[\frac{M_{2}}{r}\left(4 v_{2}^{2}-8 \mathbf{v}_{1} \cdot \mathbf{v}_{2}+2\left(\mathbf{v}_{1} \cdot \mathbf{n}\right)^{2}-4\left(\mathbf{v}_{1} \cdot \mathbf{n}\right)\left(\mathbf{v}_{2} \cdot \mathbf{n}\right)-6\left(\mathbf{v}_{2} \cdot \mathbf{n}\right)^{2}\right)\right. \\
& +\frac{M_{1}}{r}\left(\frac{5}{4} v_{2}^{2}-\frac{5}{2} \mathbf{v}_{1} \cdot \mathbf{v}_{2}-\frac{15}{4} v_{1}^{2}+\frac{39}{2}\left(\mathbf{v}_{1} \cdot \mathbf{n}\right)^{2}-39\left(\mathbf{v}_{1} \cdot \mathbf{n}\right)\left(\mathbf{v}_{2} \cdot \mathbf{n}\right)+\frac{17}{2}\left(\mathbf{v}_{2} \cdot \mathbf{n}\right)^{2}\right)
\end{aligned}
$$




$$
\begin{aligned}
& -\frac{57}{4} \frac{M_{1}^{2}}{r^{2}}-\frac{69}{2} \frac{M_{1} M_{2}}{r^{2}}-9 \frac{M_{2}^{2}}{r^{2}}-2 v_{2}^{4}+4 v_{2}^{2}\left(\mathbf{v}_{1} \cdot \mathbf{v}_{2}\right)-2\left(\mathbf{v}_{1} \cdot \mathbf{v}_{2}\right)^{2} \\
& \left.+\frac{3}{2} v_{1}^{2}\left(\mathbf{v}_{2} \cdot \mathbf{n}\right)^{2}-6\left(\mathbf{v}_{1} \cdot \mathbf{v}_{2}\right)\left(\mathbf{v}_{2} \cdot \mathbf{n}\right)^{2}+\frac{9}{2} v_{2}^{2}\left(\mathbf{v}_{2} \cdot \mathbf{n}\right)^{2}-\frac{15}{8}\left(\mathbf{v}_{2} \cdot \mathbf{n}\right)^{4}\right] \\
& +\frac{M_{2}}{r^{2}}\left(v_{1}^{i}-v_{2}^{i}\right)\left[\frac{M_{1}}{4 r}\left(55\left(\mathbf{v}_{2} \cdot \mathbf{n}\right)-63\left(\mathbf{v}_{1} \cdot \mathbf{n}\right)\right)-\frac{2 M_{2}}{r}\left(\left(\mathbf{v}_{1} \cdot \mathbf{n}\right)+\left(\mathbf{v}_{2} \cdot \mathbf{n}\right)\right)\right. \\
& +v_{1}^{2}\left(\mathbf{v}_{2} \cdot \mathbf{n}\right)+4 v_{2}^{2}\left(\mathbf{v}_{1} \cdot \mathbf{n}\right)-5 v_{2}^{2}\left(\mathbf{v}_{2} \cdot \mathbf{n}\right)-6\left(\mathbf{v}_{1} \cdot \mathbf{n}\right)\left(\mathbf{v}_{2} \cdot \mathbf{n}\right)^{2} \\
& \left.-4\left(\mathbf{v}_{1} \cdot \mathbf{n}\right)\left(\mathbf{v}_{1} \cdot \mathbf{v}_{2}\right)+4\left(\mathbf{v}_{2} \cdot \mathbf{n}\right)\left(\mathbf{v}_{1} \cdot \mathbf{v}_{2}\right)+\frac{9}{2}\left(\mathbf{v}_{2} \cdot \mathbf{n}\right)^{3}\right] .
\end{aligned}
$$

The equation of motion of body 2 can be found by interchanging $1 \rightleftharpoons 2$. These agree completely with other results for the $2 \mathrm{PN}$ equations of motion [6, 7, 12, 13, 14, 15, 16].

The remainder of this paper provides details. In Sec. II we lay out the basic equations and assumptions that underlie our method. Section III focuses on the first post-Newtonian approximation and verifies SEP to that order, while Sec. IV extends this to second post-Newtonian order. Concluding remarks are made in Sec. IV Selected detailed calculations are relegated to a series of Appendices.

\section{BASIC EQUATIONS AND ASSUMPTIONS}

We consider the motion of a binary system made up of two fluid balls of characteristic mass $m$ and size $s$. We do not assume that the bodies are spherically symmetric. They could be distorted, either because of rotation or because of tidal interactions (although we will not take either spin or tidal effects explictly into account). The only symmetry we will impose on the bodies is that each be symmetric on reflection through its center of mass. In practice this means that we will ignore any odd- $\ell$ angular harmonics in its density distribution; equivalently we will assume that the integral of any odd number of purely internal vectors (such as position or velocity relative to the center of mass) over the body vanishes.

We will treat the material making up the bodies as a perfect fluid, with the pressure required for equilibrium provided by random internal fluid motions. Thus we will break up the velocity of each fluid element in the A-th body according to $\mathbf{v}=\mathbf{v}_{A}+\overline{\mathbf{v}}$, where $\mathbf{v}_{A}$ will be a suitably defined center-of-mass velocity, and $\overline{\mathbf{v}}$ could contain both random thermal-like velocities as well as bulk internal or rotational velocities. We set the formal pressure equal to zero $(p=0)$. We also characterize the fluid by the so-called "conserved" baryon mass density, $\rho^{*}$, given by

$$
\rho^{*}=m n \sqrt{-g} u^{0},
$$

where $m$ is the rest mass per baryon, $n$ is the baryon number density, $u^{\alpha}$ is the four-velocity, and $g \equiv \operatorname{det}\left(g_{\mu \nu}\right)$ is the determinant of the spacetime metric $g_{\mu \nu}$. Henceforth, we use units in which $G=c=1$; Greek indices range over spacetime values $0,1,2,3$, while Latin indices range over spatial values $1,2,3$. From the conservation of baryon number (expressed covariantly as $\left.\nabla_{\alpha}\left(n u^{\alpha}\right)=0\right), \rho^{*}$ satisfies the exact continuity equation

$$
\frac{\partial \rho^{*}}{\partial t}+\nabla \cdot\left(\rho^{*} \mathbf{v}\right)=0
$$

where $v^{\alpha}=u^{\alpha} / u^{0}=(1, \mathbf{v})$ is the fluid's coordinate velocity, and spatial gradients and dot products use a Cartesian metric. In terms of $\rho^{*}$, the stress-energy tensor takes the form

$$
T^{\alpha \beta}=\rho^{*}(-g)^{-1 / 2} u^{0} v^{\alpha} v^{\beta} .
$$

We define the baryon rest mass, center of baryonic mass, velocity and acceleration of each body by the formulae

$$
\begin{aligned}
m_{A} & \equiv \int_{A} \rho^{*} d^{3} x, \\
\mathbf{x}_{A} & \equiv\left(1 / m_{A}\right) \int_{A} \rho^{*} \mathbf{x} d^{3} x, \\
\mathbf{v}_{A} & \equiv d \mathbf{x}_{A} / d t=\left(1 / m_{A}\right) \int_{A} \rho^{*} \mathbf{v} d^{3} x, \\
\mathbf{a}_{A} & \equiv d \mathbf{v}_{A} / d t=\left(1 / m_{A}\right) \int_{A} \rho^{*} \mathbf{a} d^{3} x,
\end{aligned}
$$


where we have used the general fact, implied by the equation of continuity for $\rho^{*}$, that

$$
\frac{\partial}{\partial t} \int \rho^{*}\left(t, \mathbf{x}^{\prime}\right) f\left(t, \mathbf{x}, \mathbf{x}^{\prime}\right) d^{3} x^{\prime}=\int \rho^{*}\left(t, \mathbf{x}^{\prime}\right)\left(\frac{\partial}{\partial t}+\mathbf{v}^{\prime} \cdot \nabla^{\prime}\right) f\left(t, \mathbf{x}, \mathbf{x}^{\prime}\right) d^{3} x^{\prime}
$$

The definitions of mass and center of mass are not unique, of course (for a review, see [3]). For example, we could have used an effective density that included internal kinetic and gravitational potential energies, as in Sec. 6.2 of [2], for example. As it turns out, our assumption that only even $\ell$ multipoles of the internal density distribution matter guarantees that all such alternative definitions actually coincide with our baryonic definition, by symmetry. Nevertheless, one could generalize our assumptions and consider the effect of different center-of-mass definitions, but that would be beyond the scope of this paper.

To $2 \mathrm{PN}$ order, the equations of hydrodynamics have the form [II, Eq. (2.23), (2.24)]

$$
d v^{i} / d t=U^{, i}+a_{P N}^{i}+a_{2 P N}^{i},
$$

where

$$
\begin{aligned}
a_{P N}^{i}= & v^{2} U^{, i}-4 v^{i} v^{j} U^{, j}-3 v^{i} \dot{U}-4 U U^{, i}+8 v^{j} V^{[i, j]}+4 \dot{V}^{i}+\frac{1}{2} \ddot{X}^{, i}+\frac{3}{2} \Phi_{1}^{, i}-\Phi_{2}^{, i}, \\
a_{2 P N}^{i}= & 4 v^{i} v^{j} v^{k} V^{j, k}+v^{2} v^{i} \dot{U}+v^{i} v^{j}\left(4 \Phi_{2}^{, j}-2 \Phi_{1}^{, j}-2 \ddot{X}^{, j}\right)-\frac{1}{2} v^{2}\left(2 \Phi_{2}^{, i}+\Phi_{1}^{, i}-\ddot{X}^{, i}\right) \\
& +v^{j} v^{k}\left(2 \Phi_{1}^{j k, i}-4 \Phi_{1}^{i j, k}+2 P_{2}^{j k, i}-4 P_{2}^{i j, k}\right)+v^{i}\left(3 \dot{\Phi}_{2}-\frac{1}{2} \dot{\Phi}_{1}-\frac{3}{2} \ddot{X}^{(3)}+4 V^{k} U^{, k}\right) \\
& +v^{j}\left(8 V_{2}^{[i, j]}-16 \Phi_{2}^{[i, j]}+4 \ddot{X}^{[i, j]}+32 G_{7}^{[i, j]}-16 U V^{[i, j]}-4 \Sigma^{, i}\left(v^{j]} v^{2}\right)+8 V^{i} U^{, j}\right. \\
& \left.-4 \dot{\Phi}_{1}^{i j}-4 \dot{P}_{2}^{i j}\right)+\frac{7}{8} \Sigma^{, i}\left(v^{4}\right)+\frac{9}{2} \Sigma^{, i}\left(v^{2} U\right)-4 \Sigma^{, i}\left(v^{j} V^{j}\right)-\frac{3}{2} \Sigma^{, i}\left(\Phi_{1}\right)-6 U_{1}^{, i}-2 \Phi_{1} U^{, i} \\
& -4 \Phi_{1}^{i j} U^{, j}+8 V^{j} V^{j, i}+4 V^{i} \dot{U}^{, i}+2 \dot{\Sigma}\left(v^{i} v^{2}\right)+4 U \Phi_{2}^{, i}+4 \Phi_{2} U^{, i}+8 U^{2} U^{, i}-\Sigma^{, i}\left(\Phi_{2}\right) \\
& +\frac{3}{2} \Sigma^{, i}\left(U^{2}\right)-2 U \ddot{X}^{, i}-2 \ddot{X}^{, i}-8 U \dot{V}^{i}-\frac{1}{2} \Sigma^{, i}(\ddot{X})+\frac{3}{4} \ddot{X}_{1}^{, i}-\frac{1}{2} \ddot{X}_{2}^{, i}+2 X^{i}+\frac{1}{24} Y^{, i} \\
& +4 \dot{V}_{2}^{i}-8 \dot{\Phi}_{2}^{i}-6 G_{1}^{, i}-4 G_{2}^{, i}+8 G_{3}^{, i}+8 G_{4}^{, i}-4 G_{6}^{, i}+16 \dot{G}_{7}^{i}-4 P_{2}^{i j} U^{, j}-4 H^{, i},
\end{aligned}
$$

where commas denote partial derivatives, overdots and the notation $(n)$ over functions denote partial time derivatives $\partial / \partial t$, parentheses and square brackets surrounding indices denote symmetrization and antisymmetrization, respectively, and repeated spatial indices are summed.

Definitions of the potentials appear in Appendix A all are defined using $\rho^{*}$. For example, $U$ is the Newtonian potential, defined by

$$
U(t, \mathbf{x}) \equiv \int_{\mathcal{M}} \frac{\rho^{*}\left(t, \mathbf{x}^{\prime}\right)}{\left|\mathbf{x}-\mathbf{x}^{\prime}\right|} d^{3} x^{\prime}
$$

where $\mathcal{M}$ is a constant-time hypersurface extending to the boundary of the near-zone of the system.

Our task is then to calculate the acceleration of, say, body \#1 through $2 \mathrm{PN}$ order, using

$$
a_{1}^{i}=\left(1 / m_{1}\right) \int_{1} \rho^{*}\left(d v^{i} / d t\right) d^{3} x
$$

At Newtonian order, the result is simple, namely

$$
\begin{aligned}
a_{1}^{i}= & -\left(1 / m_{1}\right) \int_{1} \int_{1} \rho^{*} \rho^{* \prime} \frac{\left(x-x^{\prime}\right)^{i}}{\left|\mathbf{x}-\mathbf{x}^{\prime}\right|^{3}} d^{3} x d^{3} x^{\prime} \\
& +\left(1 / m_{1}\right) \int_{1} \rho^{*} d^{3} x \int_{2} \rho^{* \prime} d^{3} x^{\prime}\left\{\nabla_{1}^{i} \frac{1}{r}+\left(\bar{x}-\bar{x}^{\prime}\right)^{j} \nabla_{1}^{i j} \frac{1}{r}+\frac{1}{2}\left(\bar{x}-\bar{x}^{\prime}\right)^{j k} \nabla_{1}^{i j k} \frac{1}{r}+\ldots\right\},
\end{aligned}
$$

where we split the position and velocity of a given point inside each body $\mathrm{A}$ according to

$$
\begin{aligned}
x^{i} & \equiv x_{A}^{i}+\bar{x}^{i}, \\
v^{i} & \equiv v_{A}^{i}+\bar{v}^{i},
\end{aligned}
$$


and expand the potential due to body \#2 in a Taylor series about the centers of mass of the two bodies. The use of multi-indices denotes products of vectorial objects; for example $x^{i j} \equiv x^{i} x^{j}, \nabla^{i j} \equiv \nabla^{i} \nabla^{j}, x^{M} \equiv x^{i_{1}} \ldots x^{i_{m}}$, and so on.

The first term in Eq. (2.10) vanishes by symmetry (Newton's third law). The first term in the second line corresponds to the normal "point-mass" Newtonian acceleration, $-m_{2} n^{i} / r^{2}$; the second term vanishes by the definition of baryonic center of mass, and the remaining terms are standard Newtonian multipole coupling terms. They depend on the size of the bodies as $s^{n}$ with $n \geq 2$. In the limit that the size of the bodies is negligible relative to their separation, the latter terms are vanishingly small; these are the kinds of terms that we will ignore throughout.

\section{EQUATIONS OF MOTION AT 1PN ORDER}

At higher PN order, there is now the possibility of correction terms proportional to inverse powers of $s$. To illustrate this, we evaluate two terms from Eq. (2.7a) explicitly:

$$
\begin{aligned}
\frac{1}{m_{1}} \int_{1} \rho^{*} v^{2} U^{, i} d^{3} x= & -\frac{1}{m_{1}} \int_{1} \rho^{*}\left(v_{1}^{2}+2 \mathbf{v}_{1} \cdot \overline{\mathbf{v}}+\bar{v}^{2}\right) d^{3} x \\
& \times\left[\int_{1} \frac{\rho^{* \prime}\left(x-x^{\prime}\right)^{i}}{\left|\mathbf{x}-\mathbf{x}^{\prime}\right|^{3}} d^{3} x^{\prime}+\frac{m_{2} x_{12}^{i}}{r^{3}}+\frac{m_{2} \bar{x}^{j}\left(\delta^{i j}-3 n^{i} n^{j}\right)}{r^{3}}+\ldots\right] \\
= & \frac{2 v_{1}^{j}}{m_{1}} \mathcal{H}_{1}^{j i}-m_{2} v_{1}^{2} \frac{x_{12}^{i}}{r^{3}}-\frac{2 \mathcal{T}_{1}}{m_{1}} \frac{m_{2} x_{12}^{i}}{r^{3}}+O\left(s^{1 / 2}\right)
\end{aligned}
$$

and

$$
\begin{aligned}
\frac{1}{m_{1}} \int_{1} \rho^{*} U U^{, i} d^{3} x= & -\frac{1}{m_{1}} \int_{1} \rho^{*} d^{3} x\left[\int_{1} \frac{\rho^{* \prime \prime}}{\left|\mathbf{x}-\mathbf{x}^{\prime \prime}\right|} d^{3} x^{\prime \prime}+\frac{m_{2}}{r}-\frac{m_{2}}{r^{3}} \overline{\mathbf{x}} \cdot \mathbf{x}_{12}+\ldots\right] \\
& \times\left[\int_{1} \frac{\rho^{* \prime}\left(x-x^{\prime}\right)^{i}}{\left|\mathbf{x}-\mathbf{x}^{\prime}\right|^{3}} d^{3} x^{\prime}+\frac{m_{2} x_{12}^{i}}{r^{3}}-\frac{m_{2} \bar{x}^{j}\left(\delta^{i j}-3 n^{i} n^{j}\right)}{r^{3}}+\ldots\right] \\
& =-\frac{\Omega_{1}^{i j}}{m_{1}} \frac{m_{2} x_{12}^{j}}{r^{3}}+2 \frac{\Omega_{1}}{m_{1}} \frac{m_{2} x_{12}^{i}}{r^{3}}-\frac{m_{2}^{2} x_{12}^{i}}{r^{4}}+O(s),
\end{aligned}
$$

where $\mathcal{T}_{1}, \Omega_{1}^{i j}, \Omega_{1}$ and $\mathcal{H}_{1}^{i j}$ are defined in Appendix B. Note that, because $\bar{v}^{2} \sim m / s$ for a body in equilibrium, the "kinetic energy" $\mathcal{T}_{1}$ scales as $1 / s$, as do the "gravitational potential energy" quantities $\Omega_{1}$ and $\Omega_{1}^{i j}$. The "gravitomagnetic" style quantity $\mathcal{H}_{1}^{i j}$ scales as $\bar{v} / s^{2} \sim 1 / s^{5 / 2}$. We have used our parity assumption to eliminate all terms that involve an integral over an odd number of internal vectors on a given body. Also, we have not kept terms that scale as positive powers of $s$. One example is a term from Eq. 3.1) proportional to $\bar{x} \bar{v} \sim s^{1 / 2}$; for rotating bodies it gives spin-dependent effects, which we treated in Papers III and IV [17, 18]. Here we ignore spin.

In the combination of $1 \mathrm{PN}$ terms $4 \dot{V}^{i}+\frac{1}{2} \ddot{X}^{, i}$ in Eq. (2.7a), the time derivatives generate accelerations inside the potentials. To the order needed for a $1 \mathrm{PN}$ calculation, we must therefore substitute the Newtonian hydrodynamic equations for those accelerations and carry out the same procedures for the integrals as described above. When we go to $2 \mathrm{PN}$ order, we will have to insert the $1 \mathrm{PN}$ hydrodynamic equations.

Carrying out these procedures for all the terms in Eq. (2.7a), and dropping terms scaling as positive powers of $s$, we obtain for the equation of motion of body 1 ,

$$
\begin{aligned}
a_{1 P N}^{i}= & -\frac{m_{2}}{r^{2}} n^{i}+\frac{m_{2}}{r^{2}} n^{i}\left[4 \frac{m_{2}}{r}+5 \frac{m_{1}}{r}-v_{1}^{2}+4 \mathbf{v}_{1} \cdot \mathbf{v}_{2}-2 v_{2}^{2}+\frac{3}{2}\left(\mathbf{v}_{2} \cdot \mathbf{n}\right)^{2}\right] \\
& +\frac{m_{2}}{r^{2}}\left(v_{1}-v_{2}\right)^{i}\left[4\left(\mathbf{v}_{1} \cdot \mathbf{n}\right)-3\left(\mathbf{v}_{2} \cdot \mathbf{n}\right)\right]-\frac{m_{2} n^{i}}{m_{1} r^{2}}\left[2 \mathcal{T}_{1}+\Omega_{1}\right] \\
& +4 \frac{m_{2} n^{j}}{m_{1} r^{2}}\left[2 \mathcal{T}_{1}^{i j}+\Omega_{1}^{i j}\right]+3 \frac{n^{j}}{r^{2}}\left[2 \mathcal{T}_{1}^{i j}+\Omega_{1}^{i j}\right]+\frac{3}{2} \frac{n^{i} n^{j} n^{k}}{r^{2}}\left[2 \mathcal{T}_{2}^{j k}+\Omega_{2}^{j k}\right] \\
& -\frac{n^{i}}{r^{2}}\left[4 \mathcal{T}_{2}+\frac{5}{2} \Omega_{2}\right]-\frac{v_{1}^{j}}{m_{1}}\left[4 \mathcal{H}_{1}^{(i j)}-3 \mathcal{K}_{1}^{i j}\right] .
\end{aligned}
$$

Note that, apart from the "point" mass terms, only terms scaling as $s^{-1}$ and $s^{-5 / 2}$ arise.

We now assume that each body is in equilibrium, implying that it is stationary, or possibly periodic on an internal dynamical timescale. As a consequence, any time derivative of the moment of inertia tensor, $I^{i j}=\int \rho^{*} \bar{x}^{i} \bar{x}^{j} d^{3} x$, can 
be set to zero or can be averaged to zero. This results in a set of virial relations derived in Appendix B which imply for each body that $2 \mathcal{T}_{A}^{i j}+\Omega_{A}^{i j}=0,2 \mathcal{T}_{A}+\Omega_{A}=0$, and $4 \mathcal{H}_{A}^{(i j)}-3 \mathcal{K}_{A}^{i j}=0$. These eliminate most terms dependent upon the structure of the bodies, leaving only the term $-n^{i} r^{-2}\left(4 \mathcal{T}_{2}+\frac{5}{2} \Omega_{2}\right)$. This cannot be eliminated by a virial relation; however we can add an arbitrary amount of $2 \mathcal{T}_{2}+\Omega_{2}=0$ to it to put it into the form

$$
-\frac{n^{i}}{r^{2}}\left[(4-2 \alpha) \mathcal{T}_{2}+\left(\frac{5}{2}-\alpha\right) \Omega_{2}\right] .
$$

Despite these terms, we can make the 1PN equations independent of any $O\left(s^{-1}\right)$ self terms by redefining the masses of each of the bodies to be,

$$
M_{A} \equiv m_{A}+(4-2 \alpha) \mathcal{T}_{A}+\left(\frac{5}{2}-\alpha\right) \Omega_{A}
$$

resulting in Eq. (1.4a). Note that the redefinition of the masses from $m_{A}$ to $M_{A}$ in the 1PN terms will affect the equations of motion only at $2 \mathrm{PN}$ order. Thus we have verified the SEP to $1 \mathrm{PN}$ order.

The choice $\alpha=3 / 2$ gives a redefined mass $M_{A}=m_{A}+\mathcal{T}_{A}+\Omega_{A}$, which naturally represents the total (baryonic plus kinetic plus gravitational) mass of the body, while the choice $\alpha=2$ gives $M_{A}=m_{A}+\frac{1}{2} \Omega_{A}$, which is the same thing, after applying the virial relation $\mathcal{T}_{A}=-\Omega_{A} / 2$, and represents rest mass plus gravitational binding energy. Either definition, when applied to an isolated gravitating system, gives the total system mass, as measured using Keplerian orbits far from the system. At 1PN order, the choice of $\alpha$ is completely arbitrary, but we will see that, to satisfy SEP at $2 \mathrm{PN}$ order, we must choose $\alpha=2$.

\section{EQUATIONS OF MOTION AT 2PN ORDER}

We now apply the same methods at $2 \mathrm{PN}$ order. We restrict attention to terms analogous to those that arose at 1PN order, namely terms that scale as $s^{-1}, s^{-5 / 2}$, as well as "point-mass" terms. The most straightforward terms to evaluate are those that involve two-body potentials and superpotentials, such as $U, V^{i}, \Phi_{1}, X$, and their various generalizations, such as $V_{2}^{i}, X^{i}, Y$, etc (see Appendix $\AA$ for definitions). We use the splitting of position and velocity as in Eq. (2.11), combined with the scaling $\bar{v} \sim s^{-1 / 2}$ and $\bar{x} \sim s$. It is a simple matter to expand and sort the terms using algebraic software. To illustrate the results, we cite a few simple examples from Eq. (2.7b):

$$
\begin{aligned}
\frac{4}{m_{1}} \int_{1} \rho^{*} v^{i} v^{j} v^{k} V^{j, k} d^{3} x= & -4 \frac{m_{2}}{r^{2}} v_{1}^{i}\left(\mathbf{v}_{\mathbf{1}} \cdot \mathbf{n}\right)\left(\mathbf{v}_{\mathbf{1}} \cdot \mathbf{v}_{\mathbf{2}}\right)-\frac{8}{m_{1}} \frac{m_{2}}{r^{2}}\left(v_{1}^{i} v_{2}^{j} n^{k} \mathcal{T}_{1}^{j k}+\left(\mathbf{v}_{\mathbf{1}} \cdot \mathbf{n}\right) v_{2}^{j} \mathcal{T}_{1}^{i j}+\left(\mathbf{v}_{\mathbf{1}} \cdot \mathbf{v}_{\mathbf{2}}\right) n^{j} \mathcal{T}_{1}^{i j}\right) \\
& +\frac{4}{m_{1}}\left(v_{1}^{i} v_{1}^{2} \mathcal{K}_{1}+v_{1}^{j} v_{1}^{2} \mathcal{H}^{i j}\right), \\
\frac{8}{m_{1}} \int_{1} \rho^{*} U^{2} U^{, i} d^{3} x= & -8 \frac{m_{2}^{3} n^{i}}{r^{4}}+16 \frac{m_{2}^{2}}{m_{1} r^{2}}\left(2 n^{i} \Omega_{1}-n^{j} \Omega_{1}^{i j}\right) .
\end{aligned}
$$

Throughout, we apply the Newtonian virial relations, $2 \mathcal{T}_{A}^{i j}+\Omega_{A}^{i j}=0$ and $4 \mathcal{H}_{A}^{(i j)}-3 \mathcal{K}_{A}^{i j}=0$ to all $2 \mathrm{PN}$ terms that scale as $s^{-1}$ and $s^{-5 / 2}$. In some terms, an additional virial relation is required, involving $d^{4} I_{A}^{i j} / d t^{4}$. An example is the term in Eq. (2.7b) involving $\partial^{4} Y^{, i} / \partial t^{4}$, where $Y$ is the superduperpotential (see Appendix $\mathrm{A}$ for definitions). Splitting $Y(\mathbf{x})$ into two terms, one from body 1 and one from body 2, one can show, using a Taylor expansion of the second term about the center of mass of body 2, that, inside body $1, Y$ is given by

$$
Y(\mathbf{x})=\int_{1} \rho^{* \prime}\left|\mathbf{x}-\mathbf{x}^{\prime}\right|^{3} d^{3} x^{\prime}+m_{2}\left|\mathbf{x}-\mathbf{x}_{2}\right|^{3}+\frac{1}{2} I_{2}^{j k} \nabla^{j k}\left|\mathbf{x}-\mathbf{x}_{2}\right|^{3}+\frac{1}{12} I_{2}^{j k l m} \nabla^{j k l m}\left|\mathbf{x}-\mathbf{x}_{2}\right|^{3}+\ldots
$$

Partial time derivatives of the terms involving the moments of body 2 either will give zero, because of our virial assumption that the moments are effectively constant in time, or will give terms proportional to the moments themselves, which therefore scale as positive powers of $s$. Thus in this case, only the contribution from body 1 and the point mass term from body 2 will contribute; no self-terms from body 2 will arise.

Next in complexity are the so-called triangle potentials, such as $P_{2}^{i j}=P\left(U^{, i} U^{, j}\right)$, and the potentials $G_{a}(a=1 . .6)$ and $G_{7}^{i}$, which depend on the field point and on two source points, hence the name triangle potentials. Luckily these can all be written in analytic form using the "triangle" function $\mathcal{G}(x A B)$, which satisfies the differential equation $\nabla^{2} \mathcal{G}(x A B)=-\left(\left|\mathbf{x}-\mathbf{x}_{A}\right|\left|\mathbf{x}-\mathbf{x}_{B}\right|\right)^{-1}$, and which is given explicitly by

$$
\mathcal{G}(x A B) \equiv-\ln \Delta(x A B)+1,
$$




$$
\Delta(x A B) \equiv\left|\mathbf{x}-\mathbf{x}_{A}\right|+\left|\mathbf{x}-\mathbf{x}_{B}\right|+\left|\mathbf{x}_{A}-\mathbf{x}_{B}\right|
$$

For example, the potential $G_{1}=P\left(\dot{U}^{2}\right)$ can be expressed in the form

$$
G_{1}=\sum_{A, B} \int_{A} \int_{B} \rho_{A}^{*} \rho_{B}^{*} d^{3} x_{A} d^{3} x_{B} v_{A}^{i} v_{B}^{j} \nabla_{A}^{i} \nabla_{B}^{j} \mathcal{G}(x A B)
$$

Consequently, terms involving triangle potentials can be evaluated with ease (using algebraic software, to be sure) using the same splitting and expansion procedure as before. For example,

$$
\begin{aligned}
-\frac{6}{m_{1}} \int_{1} \rho^{*} G_{1}^{, i} d^{3} x= & \frac{m_{2}}{r^{2}}\left\{\left[6 n^{i}\left(\mathbf{n} \cdot \mathbf{v}_{2}\right)^{2}-3 n^{i} v_{2}^{2}-3 v_{2}^{i}\left(\mathbf{n} \cdot \mathbf{v}_{2}\right)\right] \frac{m_{2}}{r}\right. \\
& \left.+\left[3 n^{i}\left(\mathbf{v}_{1} \cdot \mathbf{v}_{2}\right)-12 n^{i}\left(\mathbf{n} \cdot \mathbf{v}_{1}\right)\left(\mathbf{n} \cdot \mathbf{v}_{2}\right)+6 v_{1}^{i}\left(\mathbf{n} \cdot \mathbf{v}_{2}\right)+3 v_{2}^{i}\left(\mathbf{n} \cdot \mathbf{v}_{1}\right)\right] \frac{m_{1}}{r}\right\} \\
& +\frac{12}{m_{1}} \frac{m_{2}}{r^{2}}\left(\mathbf{n} \cdot \mathbf{v}_{2}\right)\left(v_{1}^{i} \Omega_{1}-v_{1}^{j} \Omega_{1}^{i j}\right)-6 \frac{n^{i}}{r^{2}}\left(v_{2}^{2} \Omega_{2}-v_{2}^{j} v_{2}^{k} \Omega_{2}^{j k}\right) .
\end{aligned}
$$

The most difficult term to evaluate is the final term in Eq. (2.7b), $-4 H^{, i}$. This involves the so-called "quadrangle" potential, since $H=P\left(U^{i j} P_{2}^{i j}\right)$ is a function of the field point and three source points. Unfortunately there is no known analytic formula similar to the function $\mathcal{G}$ that can be employed to simplify this potential or to expand it about the bodies' centers of mass using algebraic software. Instead an alternative and cumbersome method must be used; this is detailed in Appendix D. The result for this term is

$$
\begin{aligned}
-\frac{4}{m_{1}} \int_{1} \rho^{*} H^{, i} d^{3} x= & -\frac{m_{2} n^{i}}{r^{4}}\left(8 m_{1} m_{2}+m_{2}^{2}\right) \\
& -\frac{m_{2}}{r^{3}}\left\{\left(5 m_{1}-m_{2}\right) n^{i} \frac{\Omega_{1}}{m_{1}}+\left(5 m_{1}+m_{2}\right) n^{j} \frac{\Omega_{1}^{i j}}{m_{1}}-2\left(10 m_{1}-m_{2}\right) n^{i} n^{j} n^{k} \frac{\Omega_{1}^{j k}}{m_{1}}\right. \\
& \left.+2 n^{i} \Omega_{2}+2\left(m_{1}+3 m_{2}\right) n^{j} \frac{\Omega_{2}^{i j}}{m_{2}}-2\left(m_{1}+6 m_{2}\right) n^{i} n^{j} n^{k} \frac{\Omega_{2}^{j k}}{m_{2}}\right\} .
\end{aligned}
$$

In the limit of spherically symmetric bodies, where $\Omega_{A}^{i j}=\Omega_{A} \delta^{i j} / 3$, all the $s^{-1}$ contributions cancel, and the result agrees with that of [6].

We also must insert the $1 \mathrm{PN}$ hydrodynamical equations of motion into the accelerations that appear in the $1 \mathrm{PN}$ terms $4 \dot{V}^{i}+\frac{1}{2} \ddot{X}, i$ in Eq. (2.7a), and evaluate those $2 \mathrm{PN}$ terms. They involve only 2-body potentials, and thus are straightforward to evaluate.

Combining all the terms that scale as $s^{-1}$ and $s^{-5 / 2}$, and displaying only those terms, we obtain the result

$$
\begin{aligned}
a_{1(2 P N)}^{i}= & \frac{m_{2}}{r^{2}}\left[n ^ { i } \left\{\left(v_{1}^{2}+\frac{5}{2} \frac{m_{1}}{r}+5 \frac{m_{2}}{r}\right) \frac{\Omega_{1}}{m_{1}}+\left(v_{2}^{2}-\frac{1}{2} v_{1}^{2}+2 \mathbf{v}_{1} \cdot \mathbf{v}_{2}+\frac{3}{4}\left(\mathbf{n} \cdot \mathbf{v}_{2}\right)^{2}+4 \frac{m_{2}}{r}+13 \frac{m_{1}}{r}\right) \frac{\Omega_{2}}{m_{2}}\right.\right. \\
& \left.+\left(4 v_{2}^{k}-\frac{1}{2} v_{1}^{k}\right) v_{1}^{j} \frac{\Omega_{1}^{j k}}{m_{1}}+\left(\frac{13}{4} v_{2}^{j} v_{2}^{k}-\frac{3}{2} v_{2}^{2} n^{j} n^{k}-\frac{15}{2} \frac{m_{1}}{r} n^{j} n^{k}\right) \frac{\Omega_{2}^{j k}}{m_{2}}-\frac{9}{4} v_{2}^{j} v_{2}^{k} n^{l} n^{m} \frac{\Omega_{2}^{j k l m}}{m_{2}}\right\} \\
& -n^{j}\left\{\left(4 \mathbf{v}_{1} \cdot \mathbf{v}_{2}+20 \frac{m_{2}}{r}\right) \frac{\Omega_{1}^{i j}}{m_{1}}+\left(3 v_{2}^{2}+15 \frac{m_{1}}{r}\right) \frac{\Omega_{2}^{i j}}{m_{2}}\right\} \\
& +\left(v_{1}-v_{2}\right)^{i}\left\{\left(2 \mathbf{n} \cdot \mathbf{v}_{1}-\frac{3}{2} \mathbf{n} \cdot \mathbf{v}_{2}\right) \frac{\Omega_{2}}{m_{2}}+4 v_{1}^{j} n^{k} \frac{\Omega_{1}^{j k}}{m_{1}}\right\} \\
& +\left(4\left(\mathbf{n} \cdot \mathbf{v}_{1}\right) v_{2}^{j}-\left(\mathbf{n} \cdot \mathbf{v}_{1}\right) v_{1}^{j}-\left(\mathbf{n} \cdot \mathbf{v}_{2}\right) v_{1}^{j}\right) \frac{\Omega_{1}^{i j}}{m_{1}} \\
& \left.-9 v_{1}^{j} v_{1}^{k} n^{l} \frac{\Omega_{1}^{i j k l}}{m_{1}}-\frac{9}{2} v_{2}^{j} v_{2}^{k} n^{l} \frac{\Omega_{2}^{i j k l}}{m_{2}}-\frac{1}{m_{1}} v_{1}^{j} v_{1}^{k} v_{1}^{l}\left(\frac{15}{2} \mathcal{K}_{1}^{i j k l}-3 \mathcal{L}_{1}^{i j k l}-6 \mathcal{L}_{1}^{j i k l}\right)\right],
\end{aligned}
$$

where $\Omega_{A}^{i j k l}$, which scales as $s^{-1}$, and $\mathcal{K}_{1}^{i j k l}$ and $\mathcal{L}_{1}^{i j k l}$, which scale as $s^{-5 / 2}$, are defined in Appendix B]

We now must return to the $1 \mathrm{PN}$ equations of motion including $s^{-1}$ and $s^{-5 / 2}$ terms, Eq. (3.3), and employ virial relations that are correct to $1 \mathrm{PN}$ order. As we saw in Sec III, all self terms vanish at $1 \mathrm{PN}$ order, except for the term 
proportional to $4 \mathcal{T}_{2}+5 / 2 \Omega_{2}$. The residual term there could be absorbed into a redefinition of the masses. We must now consider the application of virial relations and the mass redefinition at higher PN order.

We first replace the term $-\left(n^{i} / r^{2}\right)\left(4 \mathcal{T}_{2}+5 / 2 \Omega_{2}\right)$ in Eq. (3.3) by the equivalent term

$$
-\frac{n^{i}}{r^{2}}\left[(4-2 \alpha) \mathcal{T}_{2}+\left(\frac{5}{2}-\alpha\right) \Omega_{2}\right]-\alpha \frac{n^{i}}{r^{2}}\left(2 \mathcal{T}_{2}+\Omega_{2}\right),
$$

and apply the $1 \mathrm{PN}$ corrected virial relations of Eqs. (B5) only to the second piece of this expression, as well as to all the other $s^{-1}$ and $s^{-5 / 2}$ terms in Eq. (3.3). The other piece of (4.8) will be absorbed into a redefinition of the mass of body 2. At the same time, we redefine all the masses in the point-mass 1PN terms using Eqs. (3.5); for those terms, to the order of approximation needed, the Newtonian virial relations may be used to simplify the renormalization to $M_{A}=m_{A}+1 / 2 \Omega_{A}$. The $s^{-1}$ and $s^{-5 / 2}$ terms generated by these substitutions cancel all the $s^{-5 / 2}$ terms and almost all the $s^{-1}$ terms in Eq. (4.7). The uncancelled $s^{-1}$ terms at $1 \mathrm{PN}$ and $2 \mathrm{PN}$ order, combined with the Newtonian acceleration, give

$$
\begin{aligned}
a_{1}^{i}(\text { self })= & -\frac{n^{i}}{r^{2}}\left[m_{2}+(4-2 \alpha) \mathcal{T}_{2}+\left(\frac{5}{2}-\alpha\right) \Omega_{2}\right. \\
& \left.+(\alpha-2) v_{2}^{2} \Omega_{2}-\frac{1}{2}(21-10 \alpha) \frac{m_{1}}{r} \Omega_{2}-\frac{1}{4}(13-6 \alpha) v_{2}^{j} v_{2}^{k} \Omega_{2}^{j k}\right] .
\end{aligned}
$$

Note that the choice $\alpha=2$ leaves the coefficient of the "Newtonian" acceleration as

$$
\begin{aligned}
m_{2}+\frac{1}{2} \Omega_{2}-\frac{1}{2} \frac{m_{1}}{r} \Omega_{2}-\frac{1}{4} v_{2}^{j} v_{2}^{k} \Omega_{2}^{j k} & =m_{2}+\frac{1}{2} \hat{\Omega}_{2} \\
& =M_{2},
\end{aligned}
$$

where

$$
\hat{\Omega}_{2}=\left(1-\frac{m_{1}}{r}\right) \Omega_{2}-\frac{1}{2} v_{2}^{j} v_{2}^{k} \Omega_{2}^{j k}
$$

is the gravitational binding energy as calculated in the local, comoving inertial frame of body 2 (see Appendix C for derivation). The quantity $M_{2}$ is precisely the total mass, comprising the baryonic mass $m_{2}$ plus the locally measured gravitational binding energy $\frac{1}{2} \hat{\Omega}_{2}$. The additional terms in (4.11) are simply the $s^{-1}$ corrections arising from the transformation from the local, comoving inertial frame to our global coordinate frame.

Thus, when all masses are written in terms of the new $M_{A}$, no $s^{-1}$ terms survive in the equations of motion, leaving only the "point"-mass terms, given by Eq. (1.4b).

\section{CONCLUSIONS}

We have studied the motion of finite sized, self-gravitationally bound, arbitarily shaped bodies at second postNewtonian order, and demonstrated that self-energy effects that scale with size of the body as $s^{-1}$ and $s^{-5 / 2}$ cancel precisely when a suitable renormalization of the masses is carried out. Since the bodies are finite, that renormalization is finite (in contrast to other, singular renormalization techniques), and consists of redefining the masses, to the PN order needed, as the sum of baryonic rest mass and gravitational binding energy, as measured in the locally comoving inertial frame of each body. We emphasize that we have made no effort to define masses or centers of mass in a covariant manner; all calculations are carried out in the harmonic coordinates that are built into our approach. We are currently investigating $2 \mathrm{PN}$ terms with other scalings, such as $s^{-7 / 2}, s^{-2}$ and so on, to see if they also cancel.

\section{Acknowledgments}

This work is supported in part by the National Science Foundation, Grant Nos. PHY 03-53180 and PHY 0652448. One of us (CMW) is grateful to the Group Gravitation Relativiste et Cosmologie (GR $\varepsilon$ CO) of the Institut d'Astrophysique de Paris for its hospitality while this work was being completed. 


\section{APPENDIX A: POTENTIALS APPEARING IN THE 2PN EQUATIONS OF MOTION}

The potentials that appear in the equations of motion are all Poisson-like potentials and their generalizations, namely a superpotential $X$ and a superduperpotential $Y$, integrated over a constant time hypersurface $\mathcal{M}$ that extends to the boundary of the near zone of the system. In the case of integrands of non-compact support, we discard all terms that depend on the radius of the near-zone, $\mathcal{R}$; see Paper I [11] for justification. All potentials are defined in terms of the conserved baryon mass density $\rho^{*}$ :

$$
\begin{aligned}
P(f) & \equiv \frac{1}{4 \pi} \int_{\mathcal{M}} \frac{f\left(t, \mathbf{x}^{\prime}\right)}{\left|\mathbf{x}-\mathbf{x}^{\prime}\right|} d^{3} x^{\prime}, \quad \nabla^{2} P(f)=-f, \\
\Sigma(f) & \equiv \int_{\mathcal{M}} \frac{\rho^{*}\left(t, \mathbf{x}^{\prime}\right) f\left(t, \mathbf{x}^{\prime}\right)}{\left|\mathbf{x}-\mathbf{x}^{\prime}\right|} d^{3} x^{\prime}=P\left(4 \pi \rho^{*} f\right), \\
X(f) & \equiv \int_{\mathcal{M}} \rho^{*}\left(t, \mathbf{x}^{\prime}\right) f\left(t, \mathbf{x}^{\prime}\right)\left|\mathbf{x}-\mathbf{x}^{\prime}\right| d^{3} x^{\prime}, \\
Y(f) & \equiv \int_{\mathcal{M}} \rho^{*}\left(t, \mathbf{x}^{\prime}\right) f\left(t, \mathbf{x}^{\prime}\right)\left|\mathbf{x}-\mathbf{x}^{\prime}\right|^{3} d^{3} x^{\prime} .
\end{aligned}
$$

The specific potentials used in the $1 \mathrm{PN}$ and $2 \mathrm{PN}$ equations of motion are then given by

$$
\begin{aligned}
U & \equiv \Sigma(1), \quad V^{i} \equiv \Sigma\left(v^{i}\right), \quad \Phi_{1}^{i j} \equiv \Sigma\left(v^{i} v^{j}\right) \\
\Phi_{1} & \equiv \Sigma\left(v^{2}\right), \quad \Phi_{2} \equiv \Sigma(U), \quad X \equiv X(1) \\
V_{2}^{i} & \equiv \Sigma\left(v^{i} U\right), \quad \Phi_{2}^{i} \equiv \Sigma\left(V^{i}\right), \quad Y \equiv Y(1) \\
X^{i} & \equiv X\left(v^{i}\right), \quad X_{1} \equiv X\left(v^{2}\right), \quad X_{2} \equiv X(U) \\
P_{2}^{i j} & \equiv P\left(U^{, i} U^{, j}\right), \quad P_{2} \equiv P_{2}^{i i}=\Phi_{2}-\frac{1}{2} U^{2} \\
G_{1} & \equiv P\left(\dot{U}^{2}\right), \quad G_{2} \equiv P(U \ddot{U}), \\
G_{3} & \equiv-P\left(\dot{U}^{, k} V^{k}\right), \quad G_{4} \equiv P\left(V^{i, j} V^{j, i}\right) \\
G_{5} & \equiv-P\left(\dot{V}^{k} U^{, k}\right), \quad G_{6} \equiv P\left(U^{, i j} \Phi_{1}^{i j}\right), \\
G_{7}^{i} & \equiv P\left(U^{, k} V^{k, i}\right)+\frac{3}{4} P\left(U^{, i} \dot{U}\right), \quad H \equiv P\left(U^{, i j} P_{2}^{i j}\right) .
\end{aligned}
$$

We refer the reader to Ref. [12], Appendix C, for further discussion of the triangle and quadrangle potentials.

\section{APPENDIX B: VIRIAL THEOREMS}

\section{Newtonian virial relations}

We assume that our bodies are in equilibrium, so that they are either stationary, or at worst periodic on an internal dynamical timescale. This implies, among other things, that any time derivative of the moment of inertia tensor, $I^{i j}=\int \rho^{*} \bar{x}^{i} \bar{x}^{j} d^{3} x$, is either zero or averages to zero. This will give us several virial relations that will simplify our equations. Considering body 1 , for example, we have that

$$
\begin{aligned}
\frac{1}{2} \dot{I}_{1}^{i j} & =\int_{1} \rho^{*} \bar{v}^{(i} \bar{x}^{j)} d^{3} x \\
\frac{1}{2} \ddot{I}_{1}^{i j} & =\int_{1} \rho^{*}\left(\bar{v}^{i} \bar{v}^{j}+\bar{x}^{(i} a^{j)}\right) d^{3} x, \\
\frac{1}{2} \dddot{I}_{1}^{i j} & =\int_{1} \rho^{*}\left(3 \bar{v}^{(i} a^{j)}+\bar{x}^{(i} \dot{a}^{j)}\right) d^{3} x,
\end{aligned}
$$

where, by virtue of the fact that $\int_{1} \rho^{*} \bar{x}^{i} d^{3} x=\int_{1} \rho^{*} \bar{v}^{i} d^{3} x=0$, we can drop the bars on the accelerations. Substituting the Newtonian equation of motion for $a^{i}$ gives

$$
\frac{1}{2} \ddot{I}_{1}^{i j}=\int_{1} \rho^{*} \bar{v}^{i} \bar{v}^{j} d^{3} \bar{x}-\int_{1} \int_{1} \rho^{*} \rho^{* \prime} \frac{\bar{x}^{(i}\left(x-x^{\prime}\right)^{j)}}{\left|\mathbf{x}-\mathbf{x}^{\prime}\right|^{3}} d^{3} x d^{3} x^{\prime}+\int_{1} \rho^{*} \bar{x}^{(i} U_{2}^{, j)} d^{3} x
$$




$$
\begin{aligned}
= & 2 \mathcal{T}_{1}^{i j}+\Omega_{1}^{i j}+O\left(s^{2}\right) \\
\frac{1}{2} \dddot{I}_{1}^{i j}= & -\int_{1} \int_{1} \rho^{*} \rho^{* \prime}\left(3 \frac{\bar{v}^{(i}\left(x-x^{\prime}\right)^{j)}}{\left|\mathbf{x}-\mathbf{x}^{\prime}\right|^{3}}+\frac{\bar{x}^{(i}\left(v-v^{\prime}\right)^{j)}}{\left|\mathbf{x}-\mathbf{x}^{\prime}\right|^{3}}-3 \frac{\bar{x}^{(i}\left(x-x^{\prime}\right)^{j)}\left(\mathbf{x}-\mathbf{x}^{\prime}\right) \cdot\left(\mathbf{v}-\mathbf{v}^{\prime}\right)}{\left|\mathbf{x}-\mathbf{x}^{\prime}\right|^{5}}\right) d^{3} x d^{3} x^{\prime} \\
& +\int_{1} \rho^{*}\left(3 \bar{v}^{(i} U_{2}^{, j)}+\bar{x}^{(i} \frac{d}{d t} U_{2}^{, j)}\right) d^{3} x \\
= & 4 \mathcal{H}_{1}^{(i j)}-3 \mathcal{K}_{1}^{i j}+O\left(s^{1 / 2}\right)
\end{aligned}
$$

where

$$
\begin{aligned}
\mathcal{T}_{1}^{i j} & \equiv \frac{1}{2} \int_{1} \rho^{*} \bar{v}^{i} \bar{v}^{j} d^{3} x \\
\mathcal{T}_{1} & \equiv \mathcal{T}_{1}^{i i}=\frac{1}{2} \int_{1} \rho^{*} \bar{v}^{2} d^{3} x \\
\Omega_{1}^{i j} & \equiv-\frac{1}{2} \int_{1} \int_{1} \rho^{*} \rho^{* \prime} \frac{\left(x-x^{\prime}\right)^{i j}}{\left|\mathbf{x}-\mathbf{x}^{\prime}\right|^{3}} d^{3} x d^{3} x^{\prime} \\
\Omega_{1} & \equiv \Omega_{1}^{i i}=-\frac{1}{2} \int_{1} \int_{1} \frac{\rho^{*} \rho^{* \prime}}{\left|\mathbf{x}-\mathbf{x}^{\prime}\right|} d^{3} x d^{3} x^{\prime}, \\
\mathcal{H}_{1}^{i j} & \equiv \int_{1} \int_{1} \rho^{*} \rho^{* \prime} \frac{v^{\prime}\left(x-x^{\prime}\right)^{j}}{\left|\mathbf{x}-\mathbf{x}^{\prime}\right|^{3}} d^{3} x d^{3} x^{\prime}, \\
\mathcal{K}_{1}^{i j} & \equiv \int_{1} \int_{1} \rho^{*} \rho^{* \prime} \frac{\left(x-x^{\prime}\right)^{i j} \mathbf{v}^{\prime} \cdot\left(\mathbf{x}-\mathbf{x}^{\prime}\right)}{\left|\mathbf{x}-\mathbf{x}^{\prime}\right|^{5}} d^{3} x d^{3} x^{\prime},
\end{aligned}
$$

and where we expanded the potential $U_{2}$ in the same manner as in Eq. (2.10), yielding only terms that scale as positive powers of $s$. (In Paper III [17], the $s^{1 / 2}$ terms in Eq. (B2b) produced some spin-orbit terms that contributed to the $1 \mathrm{PN}$ spin-orbit equations of motion.) Recall that, in these definitions, $\left(x-x^{\prime}\right)^{i j \ldots} \equiv\left(x-x^{\prime}\right)^{i}\left(x-x^{\prime}\right)^{j} \ldots$

Setting the derivatives of $I^{i j}$ to zero, we obtain the $s^{-1}$ and $s^{-5 / 2}$ virial relations for body 1 ,

$$
\begin{aligned}
2 \mathcal{T}_{1}^{i j}+\Omega_{1}^{i j} & =0, \\
4 \mathcal{H}_{1}^{(i j)}-3 \mathcal{K}_{1}^{i j} & =0 .
\end{aligned}
$$

\section{Post-Newtonian virial relations}

Working at $2 \mathrm{PN}$ order in the equations of motion requires us to obtain our virial relations correct to $1 \mathrm{PN}$ order. Accordingly we must now substitute the 1PN hydrodynamical equations into Eqs. (B1). The resulting 1PN corrections will have terms with a variety of scalings, from purely internal terms scaling as $s^{-2}$ in $\ddot{I}^{i j}$ and $s^{-7 / 2}$ in $\dddot{I}^{i j}$, to terms arising from expansion of the potentials due to body 2, also with a variety of scalings. Here we focus only on contributions scaling as $s^{-1}$ and $s^{-5 / 2}$. A straightforward calculation then yields the $1 \mathrm{PN}$ virial relations,

$$
\begin{aligned}
0= & 2 \mathcal{T}_{1}^{i j}+\Omega_{1}^{i j}-v_{1}^{2} \Omega_{1}^{i j}-\frac{3}{2} v_{1}^{k} v_{1}^{l} \Omega_{1}^{i j k l}-5 \frac{m_{2}}{r} \Omega_{1}^{i j}, \\
0= & 4 \mathcal{H}_{1}^{(i j)}-3 \mathcal{K}_{1}^{i j}+\frac{15}{2} v_{1}^{k} v_{1}^{l} \mathcal{K}_{1}^{i j k l}-9 v_{1}^{k} v_{1}^{l} \mathcal{L}_{1}^{(i j k) l} \\
& -\frac{m_{2}}{r^{2}}(\mathbf{v} \cdot \mathbf{n}) \Omega_{1}^{i j}+4 \frac{m_{2}}{r^{2}}\left(v_{1}-2 v_{2}\right)^{k} n^{(i} \Omega_{1}^{j) k}-8 \frac{m_{2}}{r^{2}} v^{(i} \Omega_{1}^{j) k} n^{k}+3 \frac{m_{2}}{r^{2}} v_{1}^{k} n^{l} \Omega_{1}^{i j k l},
\end{aligned}
$$

where $\mathbf{v} \equiv \mathbf{v}_{1}-\mathbf{v}_{2}$, and

$$
\begin{aligned}
\Omega_{1}^{i j k l} & \equiv-\frac{1}{2} \int_{1} \int_{1} \rho^{*} \rho^{* \prime} \frac{\left(x-x^{\prime}\right)^{i j k l}}{\left|\mathbf{x}-\mathbf{x}^{\prime}\right|^{5}} d^{3} x d^{3} x^{\prime} \\
\mathcal{K}_{1}^{i j k l} & \equiv \int_{1} \int_{1} \rho^{*} \rho^{* \prime} \frac{\left(x-x^{\prime}\right)^{i j k l} \mathbf{v}^{\prime} \cdot\left(\mathbf{x}-\mathbf{x}^{\prime}\right)}{\left|\mathbf{x}-\mathbf{x}^{\prime}\right|^{7}} d^{3} x d^{3} x^{\prime}, \\
\mathcal{L}_{1}^{i j k l} & \equiv \int_{1} \int_{1} \rho^{*} \rho^{* \prime} \frac{v^{\prime}\left(x-x^{\prime}\right)^{j k l}}{\left|\mathbf{x}-\mathbf{x}^{\prime}\right|^{5}} d^{3} x d^{3} x^{\prime} .
\end{aligned}
$$

The virial relations for body 2 can be obtained from these by the interchange $1 \rightleftharpoons 2$, with $\mathbf{n} \rightarrow-\mathbf{n}$. 


\section{APPENDIX C: RENORMALIZED MASS IN BODY'S REST FRAME}

We have renormalized the masses of the bodies by defining the total mass to be a sum of baryonic mass and gravitational binding energy, namely $M_{A} \equiv m_{A}+\frac{1}{2} \Omega_{A}$ modulo 2PN corrections. However, those 2PN corrections are of order $m \epsilon^{2} \sim m(m / s)^{2}$, and thus scale as $s^{-2}$. As we are only looking at $s^{-1}$ terms, we have not kept those corrections. However, there is an additional, $2 \mathrm{PN}$ order, $s^{-1}$ correction to the total mass that must be taken into account.

The baryonic mass of each body is a scalar, frame-invariant quantity, but the gravitational binding energy is not, since it depends on the size of the body. The correct, frame-invariant definition of our total mass must therefore be that mass as measured in a local inertial frame momentarily comoving with the body. Thus we define

$$
M_{A} \equiv m_{A}+\frac{1}{2} \hat{\Omega}_{A}+\delta M_{A},
$$

where $\delta M_{A}$ denotes the $2 \mathrm{PN}, s^{-2}$ corrections, which we are ignoring, and

$$
\hat{\Omega}_{A} \equiv-\frac{1}{2} \int_{A} \int_{A} \frac{\hat{\rho}^{*} \hat{\rho}^{* \prime}}{\left|\hat{\mathbf{x}}-\hat{\mathbf{x}}^{\prime}\right|} d^{3} \hat{x} d^{3} \hat{x}^{\prime}
$$

where hats denote spatial variables defined on a constant time hypersurface in the comoving frame. Now, the quantity $\hat{\rho}^{*} d^{3} \hat{x}$ is invariant, but $\left|\hat{\mathbf{x}}-\hat{\mathbf{x}}^{\prime}\right|$ is not. In Paper II, Appendix B, we showed that the transformation between spatial coordinates $\hat{x}^{i}$ in the comoving frame of body $\mathrm{A}$ and our global harmonic coordinates $x^{i}$ takes the form

$$
x^{i}=x_{A}^{i}+\hat{x}^{j}\left\{\delta_{j}^{i}+\epsilon\left(A_{j}^{i}+B_{j k}^{i} \hat{x}^{k}\right)\right\}+O\left(\epsilon^{2}\right),
$$

where $A_{j}^{i}$ and $B_{j k}^{i}$ are functions of the basis transformation from the global frame to the comoving inertial frame,

$$
\vec{e}_{\mu}=\left(\Lambda_{\mu}^{\hat{\alpha}}+\tilde{B}_{\mu \nu}^{\hat{\alpha}} \bar{x}^{\nu}\right) \vec{e}_{\hat{\alpha}},
$$

where $\bar{x}^{\nu}=x^{\nu}-x_{A}^{\nu}$. The coefficients $\Lambda_{\mu}^{\hat{\alpha}}$ correspond to boosts and coordinate rescalings, and the $\tilde{B}_{\mu \nu}^{\hat{\alpha}}$ make the frame freely falling.

Substiting Eq. (C3) into the definition of $\Omega_{1}$, we obtain

$$
\Omega_{1}=\hat{\Omega}_{1}-\epsilon A^{i j} \hat{\Omega}_{1}^{i j}+O\left(\epsilon^{2}\right) \hat{\Omega}_{1},
$$

where the term arising from $B_{j k}^{i}$ produces integrals over body 1 involving an odd number of vectors, which therefore vanish. The coefficients $A_{i j}$ arise from a boost to the velocity $v_{1}^{i}$ of body 1 , combined with a rescaling to an orthonormal basis in the field of body 2 . To the first PN order needed, it is simple to show that

$$
A^{i j}=-\frac{1}{2} v_{1}^{i} v_{1}^{j}-\frac{m_{2}}{r} \delta^{i j} .
$$

The result is, to the required order,

$$
\Omega_{1}=\hat{\Omega}_{1}+\frac{1}{2} v_{1}^{i} v_{1}^{j} \hat{\Omega}_{1}^{i j}+\frac{m_{2}}{r} \hat{\Omega}_{1} .
$$

Therefore, to $2 \mathrm{PN}$ order, and with $s^{-1}$ scaling, the total mass of each body is given by

$$
\begin{aligned}
M_{A} & =m_{A}+\frac{1}{2} \hat{\Omega}_{A}+O\left(s^{-2}\right) \\
& =m_{A}+\frac{1}{2} \Omega_{A}-\frac{1}{4} v_{A}^{i} v_{A}^{j} \Omega_{A}^{i j}-\frac{1}{2} \frac{m_{B}}{r} \Omega_{A}+O\left(s^{-2}\right),
\end{aligned}
$$

where we drop the hats on the $2 \mathrm{PN}$ terms.

\section{APPENDIX D: THE QUADRANGLE POTENTIAL $H$}

We now turn to evaluation of the term involving the quadrangle potential $H=P\left(U^{, i j} P_{2}^{i j}\right)$. This potential can be written in the form

$$
H=\frac{1}{4 \pi} \int_{\mathcal{M}} \frac{d^{3} x^{\prime}}{\left|\mathbf{x}-\mathbf{x}^{\prime}\right|} U^{, i j}\left(x^{\prime}\right) P_{2}^{i j}\left(x^{\prime}\right)
$$




$$
=\sum_{A B C} \int_{A} \rho_{A}^{*} \nabla_{A}^{i} \nabla_{A}^{j} d^{3} x_{A} \int_{B} \rho_{B}^{*} \nabla_{B}^{i} d^{3} x_{B} \int_{C} \rho_{C}^{*} \nabla_{C}^{j} d^{3} x_{C} \mathcal{H}(x A ; B C),
$$

where the function $\mathcal{H}$ of four field points is defined by

$$
\mathcal{H}(A B ; C D) \equiv \frac{1}{(4 \pi)^{2}} \int_{\mathcal{M}} \int_{\mathcal{M}} \frac{d^{3} x^{\prime} d^{3} x^{\prime \prime}}{\left|\mathbf{x}_{A}-\mathbf{x}^{\prime}\right|\left|\mathbf{x}_{B}-\mathbf{x}^{\prime}\right|\left|\mathbf{x}^{\prime}-\mathbf{x}^{\prime \prime}\right|\left|\mathbf{x}_{C}-\mathbf{x}^{\prime \prime}\right|\left|\mathbf{x}_{D}-\mathbf{x}^{\prime \prime}\right|}
$$

Unfortunately, there appears to be no closed-form analytic expression for $\mathcal{H}$ similar to Eq. (4.3) for $\mathcal{G}$. Instead, we use the first definition of $H$ in Eq. (D1), and integrate $H^{, i}$ over the mass of body 1 . After integrating once by parts, showing that the surface term at the boundary of $\mathcal{M}$ can be discarded, and using the fact that $P_{2, j}^{j k}=\frac{1}{2} \Phi_{2}^{, k}-\frac{1}{2} U U^{, k}-\Sigma\left(U^{, k}\right)$, we obtain

$$
\int_{1} \rho^{*} H^{, i} d^{3} x=\frac{1}{4 \pi} \int_{\mathcal{M}} U_{1}^{, i j} U^{, k} P_{2}^{j k} d^{3} x+\frac{1}{8 \pi} \int_{\mathcal{M}} U_{1}^{, i} U^{, k}\left[\Phi_{2}^{, k}-U U^{, k}-2 \Sigma\left(U^{, k}\right)\right] d^{3} x,
$$

where $U_{1}$ is the Newtonian potential due to body 1 only. The first term in Eq. (D3) can be expanded into the form

$$
\operatorname{Term}_{1}=\frac{1}{4 \pi} \int_{\mathcal{M}} U_{1}^{, i j}\left(U_{1}^{, k}+U_{2}^{, k}\right)\left[P_{2(11)}^{j k}+2 P_{2(12)}^{(j k)}+P_{2(22)}^{j k}\right] d^{3} x
$$

where the subscripts denote the contributions from the various bodies.

The second term in Eq. (D3) can be simplified by integrating by parts, leading to

$$
\operatorname{Term}_{2}=\frac{1}{4} \int_{1} \rho^{*}\left(U^{, i} \Phi_{2}+U \Phi_{2}^{, i}-U^{2} U^{, i}\right) d^{3} x+\frac{1}{4} \int_{\mathcal{M}} \rho^{*} U_{1}^{, i} \Phi_{2} d^{3} x-\frac{1}{4 \pi} \int_{\mathcal{M}} U_{1}^{, i} U^{, k} \Sigma\left(U^{, k}\right) d^{3} x
$$

where the first integral is only over body 1 .

To handle the integrals of noncompact support integrands, we split the domain $\mathcal{M}$ into three regions, a region $\mathcal{M}_{1}$ of radius $\mathcal{R}_{1}$ surrounding body 1 and a region $\mathcal{M}_{2}$ of radius $\mathcal{R}_{2}$ surrounding body 2 , and the remainder, $\mathcal{M}_{-} \mathcal{M}_{1}-\mathcal{M}_{2}$. To carry out the integrals, we will need suitable forms for the various potentials, $U, P_{2(11)}^{j k}, P_{2(12)}^{(j k)}$, and so on, in the appropriate regions.

This will be aided by a general expansion of the function $\mathcal{G}(A B C)$ in powers of $r_{A B} / r_{A C}$, where points $A$ and $B$ are assumed to lie inside one body, and point $C$ is inside the other body, so that $r_{A B} \sim s \ll r_{A C}$. Straightforward methods lead to the expansion

$$
\begin{aligned}
\mathcal{G}(A B C)= & -\ln r_{A C}+1-\ln 2+\frac{1}{2} \sum_{m=0}^{\infty} \frac{\left(-r_{A B}\right)^{m+1}}{(m+1) !} \\
& \times\left\{\left(n_{A B}\right)^{M} \nabla_{A}^{M}\left(\frac{1}{r_{A C}}\right)+\frac{r_{A C}}{m+1}\left(n_{A B}\right)^{M+1} \nabla_{A}^{M+1}\left(\frac{1}{r_{A C}}\right)\right\} .
\end{aligned}
$$

where $n_{A B}^{i}=x_{A B}^{i} / r_{A B}$. Then, given that

$$
P_{2(A B)}^{i j}=\int_{A} \int_{B} \rho_{A}^{*} \rho_{B}^{*} d^{3} x_{A} d^{3} x_{B} \nabla_{A}^{i} \nabla_{B}^{j} \mathcal{G}(x A B)
$$

it can be shown that, for two source points in body A and a field point outside the body,

$$
P_{2(A A)}^{i j}=\frac{1}{4} \frac{m_{A}^{2}}{y_{A}^{2}}\left(\hat{y}_{A}^{i} \hat{y}_{A}^{j}-\delta^{i j}\right)+\frac{1}{y_{A}}\left(\Omega_{A}^{i j}-\Omega_{A} \delta^{i j}\right)+O(s),
$$

where $\hat{y}_{A}^{i} \equiv\left(x-x_{A}\right)^{i} /\left|\mathbf{x}-\mathbf{x}_{A}\right|$, and $y_{A} \equiv\left|\mathbf{x}-\mathbf{x}_{A}\right|$, and $x_{A}^{i}$ now denotes the center of baryonic mass of body A. For a spherically symmetric body, this agrees with Eqs. (C6) and (C7) of Paper II [12].

Similarly, for a source point and a field point in body A, and the other source point in body B,

$$
\begin{aligned}
P_{2(A B)=}^{i j}= & \frac{m_{A} m_{B}}{2 r^{2}}\left(\delta^{i j}-2 n^{i j}\right)+\frac{m_{B}}{2 r^{2}} n^{j} X_{A}^{, i} \\
& +\frac{3}{4} \frac{m_{B}}{r^{3}} n^{<j k>}\left(2 X_{A} \delta^{i k}-\frac{1}{3} Y_{A}^{, i k}\right)+\frac{1}{4} \frac{m_{A} m_{B}}{r^{3}} \bar{x}^{k}\left(4 n^{i j k}-n^{i} \delta^{j k}-n^{k} \delta^{i j}-2 n^{j} \delta^{i k}\right)
\end{aligned}
$$




$$
\begin{aligned}
& +\frac{1}{12} \frac{m_{B}}{r^{4}} n^{<j k l>}\left(Z_{A}^{, i k l}-15 Y_{A}^{, k} \delta^{i l}\right) \\
& -\frac{1}{4} \frac{m_{B}}{r^{4}}\left(m_{A} \bar{x}^{k l}+I_{A}^{k l}\right)\left(6 n^{i j k l}-2 n^{i k} \delta^{j l}-2 n^{k l} \delta^{i j}-4 n^{j k} \delta^{i l}+\delta^{i k} \delta^{j l}\right) \\
& +O\left(s^{3}\right)
\end{aligned}
$$

where $n^{i}=\left(x_{A}^{i}-x_{B}^{i}\right) / r, r=\left|\mathbf{x}_{A}-\mathbf{x}_{B}\right|$, and $\bar{x}^{k}=x^{k}-x_{A}^{k} ; X_{A}, Y_{A}$, and $Z_{A}$ denote the superpotentials $\int_{A} \rho^{* \prime}\left|\mathbf{x}-\mathbf{x}^{\prime}\right|^{p} d^{3} x^{\prime}$ generated by body A only, where $p=1,3,5$, respectively, and $I_{A}^{k l}$ is the moment of inertia tensor of body A. Angular brackets $<>$ around indices denote the symmetric trace-free product. We have kept terms up to order $s^{2}$ in Eq. (D9) because they will ultimately be multiplied by terms that scale as negative powers of $s$.

For a source point in body A, a source point in body B and the field point between the two bodies,

$$
P_{2(A B)}^{i j}=\frac{m_{A} m_{B}}{\Delta(x A B)}\left(\frac{\left(\hat{y}_{A}-n\right)^{i}\left(\hat{y}_{B}+n\right)^{j}}{\Delta(x A B)}+\frac{\delta i j-n^{i j}}{r}\right)+O(s) .
$$

Other useful identities involving integrals over a sphere surrounding one of the bodies, say body B, include,

$$
\begin{aligned}
\frac{1}{4 \pi} \int_{0}^{\mathcal{R}_{B}} \frac{d^{3} x}{\left|\mathbf{x}-\mathbf{x}_{A}\right|\left|\mathbf{x}-\mathbf{x}_{B}\right|}= & -\frac{1}{3 \mathcal{R}_{B}} \mathbf{x}_{A} \cdot \mathbf{x}_{B}-\frac{1}{2} r_{A B}+\mathcal{R}_{B}, \\
\frac{1}{4 \pi} \int_{0}^{\mathcal{R}_{B}} \frac{x^{i} d^{3} x}{\left|\mathbf{x}-\mathbf{x}_{A}\right|\left|\mathbf{x}-\mathbf{x}_{B}\right|}= & -\frac{1}{15 \mathcal{R}_{B}}\left[x_{B}^{2} x_{A}^{i}+x_{A}^{2} x_{B}^{i}-3\left(x_{A}^{i}+x_{B}^{i}\right) \mathbf{x}_{A} \cdot \mathbf{x}_{B}\right]-\frac{1}{4}\left(x_{A}^{i}+x_{B}^{i}\right) r_{A B} \\
& +\frac{\mathcal{R}_{B}}{3}\left(x_{A}^{i}+x_{B}^{i}\right) .
\end{aligned}
$$

To illustrate the method used in evaluating $\int_{1} \rho^{*} H^{, i} d^{3} x$, we consider one integral in Eq. (D4), namely $(4 \pi)^{-1} \int_{\mathcal{M}} U_{1}^{, i j} U_{1}^{, k} P_{2(22)}^{j k} d^{3} x$. Considering first the integral over a sphere of radius $\mathcal{R}_{1}$ surrounding body 1 , we expand $P_{2(22)}^{j k}$ in powers of $\bar{x}^{m}=x^{m}-x_{1}^{m}$ about the center of mass of body 1 . The only term that gives a non-zero result that scales as $s^{0}$ or lower is the term linear in $\bar{x}$. We must therefore evaluate the integral $\int U_{1}^{i j} U_{1}^{, k} \bar{x}^{m} d^{3} x$ over a sphere surrounding body 1. This can be done using Eqs. (D11). Combining the result with $P_{2(22), m}^{j k}$ evaluated at $x_{1}$ using Eq. (D8), and keeping terms scaling as $s^{-1}$ or $\mathcal{R}_{1}^{-1}$, we obtain the term

$$
\frac{m_{2}^{2}}{4 r^{3}}\left(\Omega_{1} n^{i}-\Omega_{1}^{i j} n^{j}+2 \Omega_{1}^{j k} n^{i j k}\right)+\frac{m_{1}^{2}}{30 \mathcal{R}_{1} r^{2}}\left(\frac{7 m_{2}^{2}}{r} n^{i}+14 \Omega_{2} n^{i}-2 \Omega_{2}^{i j} n^{j}\right) .
$$

Integrating over a sphere surrounding body 2 , we expand the product $U_{1}^{, i j} U_{1}^{k}$ about $x_{2}$ in powers of $\bar{x}^{m}=x^{m}-x_{2}^{m}$. But because, inside body $2, P_{2(22)}^{j k}$ scales as $s^{-2}$, the integral $\int_{2} \bar{x}^{M} P_{2(22)}^{j k} d^{3} x$ scales as $s^{m+1}$, yielding no $s^{-1}$ or $s^{0}$ terms of interest. Finally, to integrate over the domain $\mathcal{M}-\mathcal{M}_{1}-\mathcal{M}_{2}$, we note that, because the integral over the domain $\mathcal{M}_{2}$ yields only positive power scaling, then for our purposes, we can integrate over this domain equally well by evaluating the integral over the exterior of the sphere surrounding body $1, \int_{\mathcal{R}_{1}}^{\infty} U_{1}^{, i j} U_{1}^{, k} P_{2(22)}^{j k} d^{3} x$, with the external potential $U_{1}=m_{1} / r$, and the exterior form (D8) for $P_{2(A A)}^{j k}$, but using body 2 as the source. The fact that the chosen form of $P_{2(22)}^{j k}$ is singular at $x_{2}$ does not affect the parts of the integral we are interested in, and the integral is finite. The result for this term is

$$
-\frac{7}{30 \mathcal{R}_{1}} \frac{m_{1}^{2} m_{2}^{2}}{r^{3}} n^{i}-\frac{1}{15 \mathcal{R}_{1}} \frac{m_{1}^{2}}{r^{2}}\left(7 \Omega_{2} n^{i}-\Omega_{2}^{i j} n^{j}\right)+\frac{1}{4} \frac{m_{1}^{2}}{r^{3}}\left(\Omega_{2} n^{i}-\Omega_{2}^{i j} n^{j}+2 \Omega_{2}^{j k} n^{i j k}\right) .
$$

Combining expressions (D12) and (D13), we see that the terms proportional to $\mathcal{R}_{1}^{-1}$ cancel, as they must, leaving the result for this term,

$$
\frac{1}{4} \frac{m_{1}^{2}}{r^{3}}\left(\Omega_{2} n^{i}-\Omega_{2}^{i j} n^{j}+2 \Omega_{2}^{j k} n^{i j k}\right)+\frac{1}{4} \frac{m_{2}^{2}}{r^{3}}\left(\Omega_{1} n^{i}-\Omega_{1}^{i j} n^{j}+2 \Omega_{1}^{j k} n^{i j k}\right) .
$$

This happens to be antisymmetric on interchange of 1 with 2 (whereby $n^{i} \rightarrow-n^{i}$ ), which is to be expected, since this particular term can also be written in the form

$$
\frac{1}{2(4 \pi)^{2}} \int_{\mathcal{M}} U_{1}^{, j} U_{1}^{, k} U_{2}^{, j^{\prime}} U_{2}^{, k^{\prime}} \frac{d^{3} x d^{3} x^{\prime}\left(x-x^{\prime}\right)^{i}}{\left|\mathbf{x}-\mathbf{x}^{\prime}\right|^{3}}
$$


which is manifestly antisymmetric under $1 \rightleftharpoons 2$. The remaining contributions to Term ${ }_{1}$ in Eq. (D4) and Term in Eq. $^{2}$. (D5) can be evaluated in the same manner. The final result for terms scaling as $s^{-1}$, together with the point-mass contributions, is given by Eq. (4.6).

[1] C. M. Will and K. Nordtvedt, Jr., Astrophys. J. 177, 757 (1972).

[2] C. M. Will, Theory and Experiment in Gravitational Physics (Cambridge University Press, Cambridge, 1993).

[3] T. Damour, in 300 Years of Gravitation, edited by S. W. Hawking and W. Israel (Cambridge University Press, London, 1987), p. 128.

[4] C. M. Will, Living Rev. Relativity 9, 3 (2006) [Online article]: cited on 1 May 2007, www.livingreviews.org/lrr-2006-3.

[5] R. A. Breuer and E. Rudolph, Gen. Relativ. Gravit. 14, 181 (1982).

[6] S. M. Kopeikin, Sov. Astron. 29, 516 (1985).

[7] L. P. Grishchuk and S. M. Kopeikin, In Relativity in Celestial Mechanics and Astrometry, edited by J. Kovalevsky and V. A. Brumberg (Reidel, Dordrecht, 1986), p. 19.

[8] L. Blanchet and G. Faye, J. Math. Phys. 41, 7675 (2000).

[9] L. Blanchet and G. Faye, J. Math. Phys. 42, 4391 (2001).

[10] Y. Itoh, T. Futamase, and H. Asada, Phys. Rev. D 62, 064002 (2000).

[11] M. E. Pati and C. M. Will, Phys. Rev. D 62, 124015 (2000).

[12] M. E. Pati and C. M. Will, Phys. Rev. D 65, 104008 (2002).

[13] T. Damour and N. Deruelle, Phys. Lett. 87A, 81 (1981).

[14] G. Schäfer and N. Wex, Phys. Lett. A 174, 196 (1993).

[15] L. Blanchet, G. Faye and B. Ponsot, Phys. Rev. D 58, 124002 (1998).

[16] Y. Itoh, T. Futamase, and H. Asada, Phys. Rev. D 63, 064038 (2001).

[17] C. M. Will, Phys. Rev. D 71, 084027 (2005).

[18] H. Wang and C. M. Will, Phys. Rev. D 75, 064017 (2007). 